RUNNING HEAD: COACHING EFFECTIVENESS

Coaching Effectiveness: The Coach-Athlete Relationship at its Heart

Sophia Jowett

School of Sport, Exercise and Health Sciences

Loughborough University

Current Opinion in Psychology 2017, 16:154-158 This review comes from a themed issue on Sport psychology Edited by Peter Beek, Vana Hutter and Raoul Oudejans http://dx.doi.org/10.1016/j.copsyc.2017.05.006 2352-250X/Crown Copyright ã 2017 Published by Elsevier Ltd. All rights reserved. 


\begin{abstract}
Coaching has been often viewed as a context within which coaches operate to largely bring about changes in athlete's performance and wellbeing. One key factor to successful outcomes in coaching is the quality of the relationship between coaches and athletes. In this article, I propose that the coach-athlete relationship is at the heart of coaching. Moreover, the aim is to describe and explain how the quality of the relationship coaches and athletes develop and maintain over the course of their sporting partnership alongside coaches and athletes' knowledge and outcomes, form a system that is capable of defining coaching effectiveness and success.
\end{abstract}

Keywords: coaching effectiveness, coach-athlete relationships 


\section{Coaching Effectiveness: The Coach-Athlete Relationship at its Heart}

At its simplest form, coaching concerns two people: the coach and the athlete. These two people form a unique dyadic relationship that holds a great deal of power and allows its members to achieve their individual and relationship goals. There is abundant anecdotal and empirical evidence to highlight that neither the coach nor the athlete can "do it alone"; they both need one another to achieve in sport [1]. When coaching is viewed as either athlete-centred or coach-centred [2, 3] -, its scope, quality and functions become restricted, whereas, when coaching is viewed as coach-athlete-centred, its scope becomes readily inclusive and mutually empowering. A coach-athlete-centred approach supplies a solid basis from which to understand not only the entire process and practice of coaching but also its effectiveness. In other words, the effectiveness and success of coaching reside within the coach and the athlete and the unit relationship they develop. Within this conceptualisation the coach and the athlete need one another to develop, grow and succeed (however one defines success: satisfaction, skill development or performance success, win/loss records). Hence, the emphasis is placed on the genuine purpose and positive intent of the coach-athlete relationship. The relationship becomes the medium that motivates, assures, satisfies, comforts, and supports coaches and athletes to enhance their sport experience, performance, and well-being [4].

\section{The current thinking of sport coaching}

Over the years, researchers from diverse disciplines including pedagogy, sociology, philosophy, and psychology have attempted to define and conceptualise sport coaching and coaching effectiveness $[5,6,7,8]$. While these attempts have been 
somewhat helpful in terms of capturing the breadth, diversity and prospect, they often lack clarity and specificity. Conceptualisations of coaching that are not accompanied by clear and specific operationalisations are strikingly complex to readily quantify and reliably measure. Nonetheless, measurement is a necessary foundation for social research and as such "gathering data without ... conscientious efforts to operationalise key concepts often is a wasted effort" [9]. In fact, the inadequacy of the various conceptualisations of coaching has been acknowledged by many scientists working in the broad field of sport coaching [10] .

While the scope of this article is not to review the various conceptualisations of coaching, I will briefly refer to four approaches that have attempted to describe and define it over the past 15 years or so. On one hand, Bowes and Jones [11] explained that coaching is a complex system within which coaches work on the "edge of chaos" (p. 235), negotiating peculiarities, intricacies and ambiguities. While more recently, Jones and colleagues [7] proposed that the complexities of coaching can be managed or "orchestrated". Accordingly, the notion of orchestration brings a sense of order through such coaching interpersonal behaviours as engaging, interacting, communicating, perspective taking, empathising, reflecting, empowering, collaborating, trusting, and understanding to name a few [7]. Both these approaches emphasise the chaos and order all at the same time, though its extensive breadth may currently stand against it, in terms of providing conceptual and operational frameworks from which empirical research can generate a body of knowledge that is organised and systematic.

On the other hand, attempts to capture the concept of coaching in its entirety may have been stimulated from work initially conducted by Lyle [8] as well as Potrac and colleagues [12] and subsequently others [13, 14]. For example, Lyle [8] describes 
coaching as a "process ... dependent on the integration of the whole being greater than the sum of its parts" (p. 97). While this description underlines the multifaceted nature of coaching, it does not spell out the facets that contribute to the integrated or holistic nature of coaching. The difficulty in endeavouring to capture all the parts of coaching in a manner that is holistic may simply be unattainable for a concept that has been characterised as chaotic as well as ambiguous and uncertain [11] . Cassidy [15] explained that despite the complexity of the notion of holistic coaching, it is important to consider. In her account, she proposed an alternative framework where socio-cultural [16] and psycho-social [17] dimensions were bridged in an effort to better understand the current elusiveness and vagueness of holistic coaching.

Considering the extant coaching literature, Côté and Gilbert [18] offered an integrating conceptualisation within which coaches' knowledge and athletes' outcomes delineated coaching effectiveness. Coaches' knowledge was divided into professional or specialised knowledge for sport, interpersonal knowledge for connecting appropriately and effectively with others (e.g., athletes, coaches, support staff, parents), and intrapersonal knowledge for self-reflection and self-awareness allowing continued learning. Athletes' outcomes were understood in terms of athletes' technical, tactical, performance skills (competence), positive self-worth (confidence), ability to connect with others (connection) and display respect, integrity and responsibility (character). Although knowledge and outcomes were central to this integrative conceptualisation of coaching, contextual factors were also important. Both, the performance level and developmental issues within which coaches and athletes operated were thought to define whether the coaching context was participation-focused (recreational, developmental) or performance-focused (elite) $[8,18]$. Accordingly, coaches' knowledge and athletes' outcomes were thought to be 
determined by the context, making all these three aspects important in the evaluation of coaching effectiveness.

Coaching is evidently conceptualised and understood through different approaches or frameworks and four of them briefly discussed: chaos, orchestration (order), holism, and/or integration. Whatever approach one chooses to utilise, the focus is or should be on the coach and the athlete. Coaches and athletes are inseparable entities within the context of coaching whether it is participation or performance. Although the conceptualisations discussed above may have placed more or less emphasis on the coaches' knowing, doing and/or being, none of these notions can be considered in isolation from athletes' knowing, doing and/or being. Coaching is an interpersonal process [8] where both a coach and an athlete inevitably engage with one another and thus effective coaching could be more readily understood through the quality of the connections coaches and athletes develop. The quality of the relationship may more easily allow gaining insights into what goes on between coaches and athletes. Such an approach may then facilitate descriptions regarding what their partnership is like (how do they relate, connect, bond) and in turn explanations about why they act and interact in the way they do.

For example, research by Nash and her colleagues [19] explained that both longterm and all-rounded development of the athlete is a central aspect of coaching excellence. They further explained that expert coaches, plan and execute training sessions with the individual athletes' needs in mind. This can only be achieved successfully if indeed coaches and athletes connect in ways that allow them to trust and commit to, as well as know and understand one another. Thus, in this paper, I propose that the quality of the coach-athlete relationship describes and defines the essence of coaching, its effectiveness and success and, in turn, more accurately 
captures the interplay of coaches' knowledge, athletes' outcomes and the coaching context (participation versus performance). Fuelled by communication (verbal and/or non-verbal), the quality of the coach-athlete relationship can be a powerful vehicle for both coaches and athletes' long-term development, personal growth and transformation [1].

\section{The coach-athlete relationship at the heart of coaching}

The coach-athlete relationship is defined as a social situation [1]. This social situation is continuously shaped by interpersonal thoughts, feelings and behaviours of the coach and the athlete. The definition further explains that a coach and an athlete are mutually and causally interdependent and thus how one feels, thinks and behaves affects and is affected by how the other feels, thinks and behaves. Jowett and Shanmugam [1] described the operational model of the quality of the relationship as follows:

- Closeness reflects interpersonal feelings of coaches and athletes that largely encapsulate an affective bond through their mutual respect, trust, appreciation, and liking for one another

- Commitment reflects interpersonal thoughts of coaches and athletes of maintaining a close (as opposed to distant, detached, unfriendly) relationship over time despite "ups and downs"

- Complementarity reflects coaches and athletes' interpersonal behaviours of leadership (reciprocal complementarity) and co-operation (corresponding complementarity). 
- Co-orientation reflects coaches and athletes' level of interdependence in terms of similarity and understanding concerning their views of the quality of their relationship

The 4 Cs provide operational meaning to the quality of the coach-athlete relationship. Accordingly, the quality of the relationship is viewed as a medium that allows coaches and athletes to express their wishes and fulfil their ambitions [1]. For example, in good quality relationships coaches and athletes invest time, effort and energy to achieve goals and priorities they have agreed on. In contrast, coaches and athletes in poor quality relationships may lack the commitment and desire to pursue ambitious objectives, are unwilling to work with each other to develop physical skills (e.g., new techniques), to overcome difficulties (e.g., injury) and in turn achieve important outcomes together. Subsequently, the quality of the relationship can function as a barometer of coaching effectiveness. When the quality of the relationship is good, coaching benefits because the relationship contains active ingredients (e.g., respect, trust, commitment, collaboration) that are important for positive and mutual influence. However, when the quality of the relationship is poor coaching suffers because the relationship is absent of active ingredients that allow the coaches and athletes to care for, commit to, co-operate with one another.

\section{Measurement and Research}

There have been a series of qualitative studies exploring the content and functions of relationships that were successful and less so $[20,21,22,23,24,25]$. Overall, the findings highlight that the quality of the coach-athlete relationship as defined and operationalised by the $4 \mathrm{Cs}$ is instrumental to performance success and wellbeing. The development and validation of Coach-Athlete Relationship Questionnaires [26, 
$27,28,29]$ facilitated the measurement of closeness, commitment and complementarity from both direct (one's own viewpoint) and meta-perspectives (one's own view of the other's viewpoint). These psychometrically validated tools enabled to readily explore antecedent and consequent variables of the coach-athlete relationship. In a nutshell, this research highlights that gender is associated with the quality of the relationship. Same gender coach-athlete dyads appear to perceive higher levels of quality relationship as defined by closeness, commitment and complementarity than their other gender coach-athlete dyads [30]. The similarityattraction theory suggests individuals like and are attracted to others who are similar, rather than dissimilar, to themselves; "birds of a feather, flock together" the adage goes [31]. Coaches and athletes of the same gender may feel they have something in common that connects them - of course this level of assumed similarity may be achieved in other gender relationships through identifying common grounds (shared interests, goals, beliefs, or opinions, preferences). Moreover, athletes' personality has shown to affect coaches' perceptions of the quality of the relationship [29, 32]. For example, coaches of athletes who have a less desirable or potentially more difficult personality (e.g., anxious or avoidant attachment styles or neuroticism) are more likely to think of the quality of the coach-athlete relationship as less positive and thus experience lower levels of closeness, commitment, and complementarity. Research also suggests that athletes with good quality coach-athlete relationships receive better coaching from their coaches [33]. Last but not least, athletes and coaches who believe that the coach-athlete relationship is mutually trusting, respective, appreciative (closeness), and committed as well as co-operative, responsive, friendly, comfortable, and relaxed (complementarity) are more likely to be satisfied with performance, personal treatment, training and instruction [30], 
experience higher levels of motivation [34] and physical self-concept [35], as well as enjoy higher levels of team cohesion and collective efficacy [36, 37].

\section{Coach-athlete relationship, coach leadership and behaviour}

Vella and colleagues [38] in a review paper underlined that while coach leadership models $[39,40,41]$ were formulated and extensively tested in sport "serving their intended purpose, they are confusing" (p. 426). Thus, Vella et al. [38] proposed that "coach leadership is not purely a behavioural process, but it is also a process of interpersonal influence that includes interpersonal variables relating to the coach-athlete relationship" (p. 431). Just like coach leadership models, motivational theoretical frameworks attached significance to the coach-athlete relationship [42]. These motivational frameworks view the relationship through the lenses of coach behaviours alone, postulating that coaches' autonomy supportive behaviours and controlling behaviours for example influence athletes' motivation [42]. More, recently research has indicated that coach-athlete relationships and coach leadership as well as coach-athlete relationships and coach behaviours when considered together are better predictors of athletes' and coaches' outcomes [43, 44, 45].

Currently, it is not clear how leadership (behaviours) and relationship constructs may interact. It may be that the coach-athlete relationship provides an opportunity for coaches to develop better leadership (behaviours). The relationship may serve as a medium through which coaches adjust and adapt by considering the other (athlete) in the relationship. As mentioned earlier, coaches and athletes inevitably engage with one another; they simply don't work in isolation and so they need to work together. Relationships may function as the building blocks for organising activities in order to achieve important goals together. And it may be that through these relationships, coaches are empowered to turn their athletes into leaders themselves. 
Overall, while the coach-athlete relationship is proposed to be at the heart of coaching, we need to start considering the role of coach leadership and behaviours and the inclusion of other key interpersonal constructs including communication [46] as well as conflict [47] within the broader conceptual landscape of coaching.

\section{Concluding Remarks}

Coaching is a notion that has been challenged by many researchers. The net result has been a proliferation of definitions of coaching and coaching frameworks. This paper argues that coaches and athletes are locked into a two-person (dyadic) relationship and its quality can offer a measure of coaching and its effectiveness. Figure 1 depicts a system of coaching effectiveness where the coach and the athlete are placed at its heart whilst coaches and athletes' knowledge and outcomes are important interconnected factors. Even though coaches and athletes begin the journey of sport with a degree of (more or less) knowledge, over time it is the working relationship that is likely to determine what knowledge is required to bring about important outcomes such as improved skill and enhanced performance as well as increased satisfaction, happiness and wellbeing. The relationship is instrumental because it can activate important processes of coaching such as influencing, supporting, helping, guiding, instructing as well as listening, willing, following, accepting, and so on in order for both coaches and athletes to develop, grow, achieve and succeed. It is the unity of these two people that powers and empowers the entire coaching system as seen in Figure 1. Ultimately, coaches and athletes share the power through their dyadic relationship for the greater good. However, without quality coach-athlete relationships, there can be no effective or purposeful coaching. This model brings together theoretical frameworks and attempts to provide conceptual and operational clarity; it may provide the impetus for more theoretical 
and empirical research, generating systematic knowledge that can be more readily used in coaching practice and education.

\section{Highlights}

- The essence of coaching effectiveness has yet to be agreed and captured

- Proliferation of coaching conceptualisations limits its systematic study

- Coaching effectiveness hinges on quality coach-athlete relationships

- A solution-focused, system model offers new possibilities for research and practice 


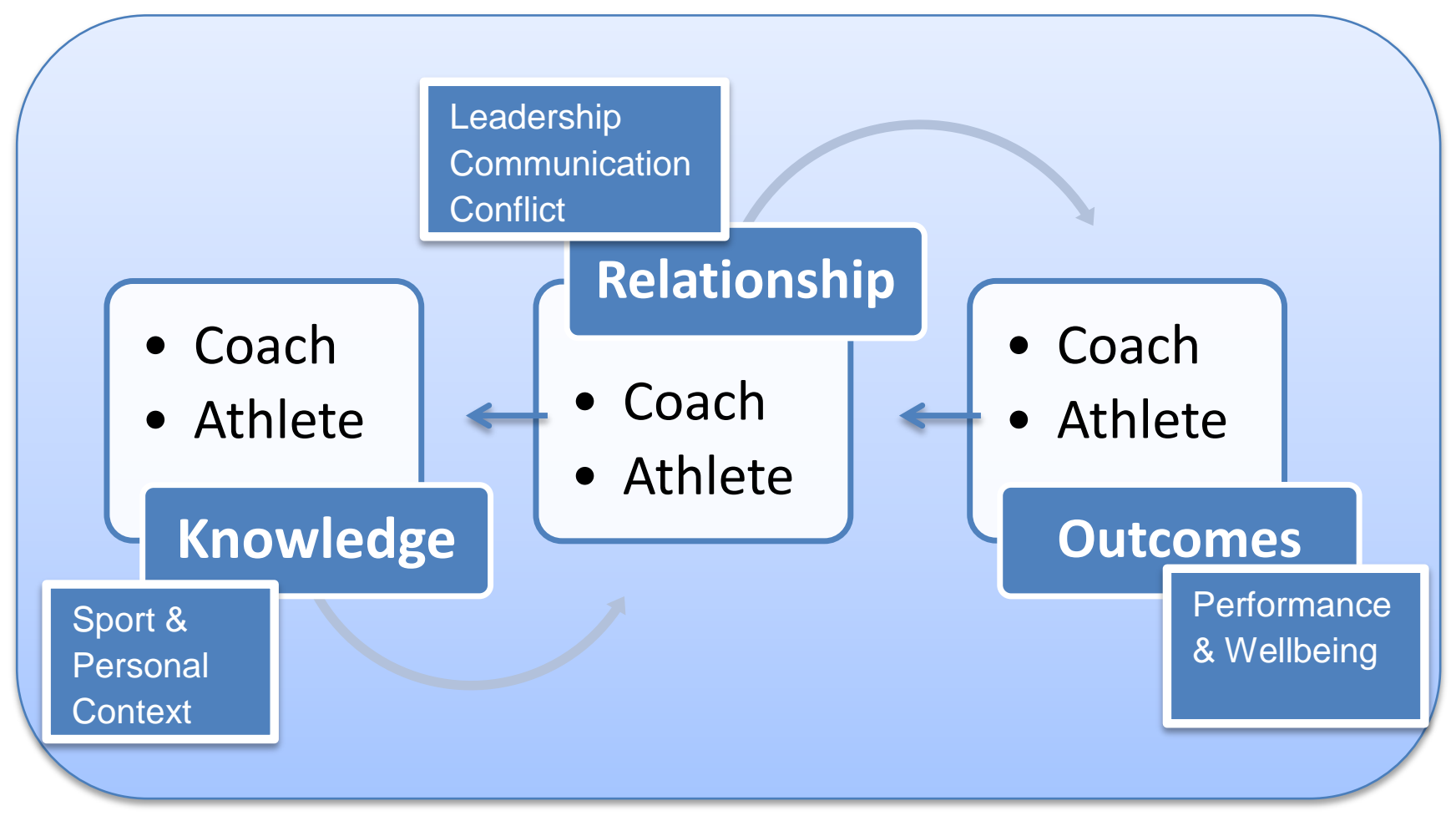

Figure 1. A system of coaching effectiveness that incorporates the coach and the athlete at every step 


\section{References}

1. **Jowett, S., \& Shanmugam, V. (2016). Relational Coaching in Sport: Its psychological underpinnings and practical effectiveness. In R. Schinke, K.R. McGannon, \& B. Smith (Eds), Routledge International Handbook of Sport Psychology (pp. 471-484). Routledge.

A review of the current literature revolving around the $3+1 \mathrm{Cs}$ coachathlete relationship model and the important role communication plays for coaches

2. Cassidy, T., \& Kidman, L. (2010). Initiating a national coaching curriculum: A paradigmatic shift? Physical Education and Sport Pedagogy, 15 (3), 307-322.

3. Kidman, L., \& Davis, W. (2006), Empowerment in Coaching, In J. Broadhead and W. Davis (Eds), Ecological Task Analysis Perspectives on Movement, Champaign, IL: Human Kinetics.

4. Jowett, S., O'Broin, A., \& Palmer, S. (2010). On understanding the role and significance of a key two-person relationship in sport and executive coaching. Sport \& Exercise Psychology Review, 6(2), 19-30.

5. Côté, J., Salmela, J., Trudel, P., Baria, A. \& Russell, S. (1995). The coaching model; a grounded assessment of expert gymnastic coaches knowledge, Journal of Sport and Exercise Psychology, 17(1), 1-17.

6. Horn, T.S. (2008). Coaching effectiveness in the sport domain. In T.S. Horn (Ed.), Advances in Sport Psychology, Champaign, IL: Human Kinetics.

7. *Jones, R. L., Bailey, J., \& Thompson, I. (2013). Ambiguity, noticing, and orchestration: Further thoughts on managing the complex coaching context. In P. Potrac, W. Gilbert, \& J. Denison (Eds.), The Routledge handbook of sports coaching (pp. 271-283). London: Routledge.

It provides a perspective on how the "chaos" or complexity of coaching can be "orchestrated" organised and managed

8. ' 'Lyle, J. (2002). Sports coaching concepts: A framework for coaches' behaviour. London: Routledge.

A classic authored booked that provides a solid grounding around coaching issues

9. Chambliss, D.F., \& Schutt, R.K. (2013). Making sense of the social world: Methods of Investigation. London: Sage.

10. Jones, R. L. \& Wallace, M. (2005) Another bad day at the training ground: coping with ambiguity in the coaching context. Sport, Education and Society, 10(1), 119-134.

11. Bowes, I., \& Jones, R. (2006). Working at the edge of chaos: Understanding coaching as a complex interpersonal system. The Sport Psychologist, 20, 235-245.

12. Potrac, P., Jones, R., \& Armour, K. (2002). It's about getting respect: The coaching behaviours of a top-level English football coach. Sport, Education and Society, 7 (2), 183-202.

13. Abraham, A., Collins, D., \& Martindale, R. (2006). The coaching schematic: Validation through expert coach consensus. Journal of Sports Sciences, 24 (6), 549-564.

14. Cushion, C., Armour, K., \& Jones, R.L. (2006). Locating the coaching process in practice models "for" and "of" coaching. Physical Education Sport Pedagogy, 11 (1), 83-99. 
15. ${ }^{*}$ Cassidy, T. (2013). Holistic sports coaching: A critical essay. In P. Potrac, W. Gilbert, \& J. Denison (Eds.), Routledge Handbook of Sports Coaching (pp. 172-183). New York, NY: Routledge.

A critique of the challenges associated with viewing sports coaching from a holistic approach

16. Denison, J. (2007). Social theory for coaches: A Foucauldian reading of one athlete's poor performance. International Journal of Sport Science and Coaching, 2 (4), 369-383.

17. Mallett, C. \& Rynne, S. (2010). Holism in sports coaching: Beyond humanistic psychology: A commentary. International Journal of Sport Science and Coaching, 5 (4), 453-457.

18. ${ }^{* *}$ Côté, J., \& Gilbert, W., (2009). An Integrative Definition of Coaching Effectiveness and Expertise, International Journal of Sports Science and Coaching, 4, 307-323.

It provides an integrative account of coaching effectiveness and introduces a new conceptualisation, supplies examples and discusses ways forward

19. *Nash, C., S., Sproule, J., \& Horton, P. (2011). Excellence in coaching: The art of and skill of elite practitioners. Research Quarterly for Exercise and Sports, 82 (2), 229-238.

This is a study that outlines what makes coaches exceptionally good, in fact elite and excellent.

20. Jowett, S. (2003). When the honeymoon is over: A case study of a coach athlete relationship in crisis. The Sport Psychologist, 17, 444-460.

21. Jowett, S. (2008b). Outgrowing the familial coach - athlete relationship. International Journal of Sport Psychology, 39, 20-40.

22. Jowett, S., \& Carpenter, P. (2015). The concept of rules in the coach-athlete relationship. Sports Coaching Review. 4, 1-23.

23. Jowett, S., \& Cockerill, I.M. (2003). Olympic Medallists' perspective of the athlete - coach relationship. Psychology of Sport and Exercise, 4, 313-331.

24. Jowett, S., \& Frost, T.C. (2007). Race/Ethnicity in the all-male coach-athlete relationship: Black footballers' narratives. Journal of International Sport and Exercise Psychology. 3, 255-269.

25. Jowett, S., Timson-Katchis, M., \& Adams, R. (2007). Too close for comfort? Dependence in the dual role of parent/coach-child/athlete relationship. International Journal of Coaching Science, 1, 59-78.

26. Jowett, S. (2009). Factor Structure and Criterion Validity of the MetaPerspective Version of the Coach-Athlete Relationship Questionnaire (CARTQ). Group Dynamics: Theory, Research and Practice, 13, 163-177.

27. Jowett, S., \& Ntoumanis, N. (2004). The Coach - Athlete Relationship Questionnaire (CART - Q): Development and initial validation. Scandinavian Journal of Medicine and Science in Sports, 14, 245-257.

28. Yang, X. S., \& Jowett, S. (2013). Conceptual and Measurement Issues of the Compelementarity Dimension of the Coach-Athlete Relationship Across Cultures. Psychology of Sport \& Exercise. 14, 830-841

29. Yang, X. S., Jowett, S., \& Chan, D.K. (2015). Effects of the Big-Five Personality Traits on the Quality of Relationship and Satisfaction in CoachAthlete Dyads. Scandinavian Journal of Medicine and Sports Sciences, 25 (4), 568-580. 
30. Jowett, S., \& Nezlek, J. (2012). Relationship Interdependence and Satisfaction with Important Outcomes in Coach-Athlete Dyads. Journal of Social and Personal Relationships, 29, 287-301.

31. Byrne, D., Clore, G. L., \& Smeaton, G. (1986). The attraction hypothesis: Do similar attitudes affect anything? Journal of Personality and Social Psychology, 51, 1167-1170.

32. Davis, L., \& Jowett, S. (2013). Attachment Styles Within the Coach-Athlete Dyad: Preliminary Investigation and Assessment Development. Journal of Clinical Sport Psychology, 7, 120-145.

33. Jowett, S., Michel, N., \& Yang, S. (2016). Coaching Relationships and Coaching Behaviours: What is the link? Manuscript under review.

34. Adie, J., \& Jowett, S. (2010). Athletes' meta-perceptions of the coach-athlete relationship, multiple achievement goals and intrinsic motivation among track and field athletes. Journal of Applied Social Psychology, 40, 2750-2773.

35. Jowett, S. (2008a). Moderators and mediators of the association between the coach-athlete relationship and physical self-concept. International Journal of Coaching Science, 2, 43-62.

36. Jowett, S., Shanmugam, V., \& Caccoulis, S. (2012). Collective efficacy as a mediator of the link between interpersonal relationships and athlete satisfaction in team sports. International Journal of Sport and Exercise Psychology, 10, 66-78.

37. Hampson, R. \& Jowett, S. (2014).Effects of Coach Leadership and CoachAthlete Relationship on Collective Efficacy. Scandinavian Journal of Medicine and Sport Sciences, 24(2), 454-460.

38. *Vella, S., Oades, L. G. \& Crowe, T. P. (2010). The application of coach leadership models to coaching practice: Current state and future directions. International Journal of Sports Science and Coaching, 5 (3), 425-434.

It provides an alternative thinking or an expanded view about coach leadership and coach-athlete relationship and their potential associations

39. Chelladurai, P., \& Riemer, H.A. (1998). Measurement of Leadership in Sport, in: Duda, J.L., ed., Advances in Sport and Exercise Psychology Measurement, Fitness Information Technology, Morgantown, WV, 1998, 227-256.

40. Smith, R.E., \& Smoll, F.L. (2007). Social-Cognitive Approach to Coaching Behaviors. In S. Jowett \& D. Lavallee. (Eds.), Social Psychology in Sport (pp. 75-90). Champaign, IL: Human Kinetics.

41. Callow, N., Smith, M.J., Hardy, L., Arthur, C.A., \& Hardy, J., (2009). Measurement of Transformational Leadership and It's Relationship with Team Cohesion and Performance Level, Journal of Applied Sport Psychology, 21, 395-412.

42. Mageau, G. A., \& Vallerand, R. J. (2003). The coach-athlete relationship: A motivational model. Journal of Sports Sciences, 21(11), 883-904.

43. Felton, L., \& Jowett, S. (2013). "What do coaches do" and "how do they relate": Their effects on athletes' psychological needs and functioning. Scandinavian Journal of Medicine and Sports Sciences, 23, 130-139.

44. Jowett, S., \& Chaundy, V. (2004). An investigation into the impact of coach leadership and coach-athlete relationship on group cohesion. Group Dynamics: Theory, Research and Practice, 8, 302-311. 
45. Vella, S. A., Oades, L. G. \& Crowe, T. P. (2013). The relationship between coach leadership, the coach-athlete relationship, team success, and the positive developmental experiences of adolescent soccer players. Physical Education and Sport Pedagogy, 18 (5), 549-561.

46. Rhind, D., \& Jowett, S. (2011). Working with coach-athlete relationships: Their quality and maintenance. In S. Mellalieu \& S. Hanton (Eds.), Professional Practice in Sport Psychology: A Review (219-248). Routledge.

47. Wachsmuth, S., Jowett, S., \& Harwood, C. (2017). Conflict among athletes and their coaches: what is the theory and research so far? International Review of Sport and Exercise Psychology, 10 (1), 84-107. 